\title{
Rethinking the ethics of research involving nonhuman animals: introduction
}

\author{
Tom L. Beauchamp • Hope R. Ferdowsian • \\ John P. Gluck
}

Published online: 3 April 2014

(C) Springer Science+Business Media Dordrecht 2014

In the relatively short time since 2006-when Theoretical Medicine and Bioethics published an issue on moral issues relevant to the use of nonhuman animals in research [1] - significant changes have occurred for nonhuman animals in many quarters. Public sentiment, new policy initiatives, and scientific studies of nonhuman animals' capacities have all influenced the ways in which nonhuman animals are perceived and treated in research. Today, a large body of information is available for use in decision making about the acceptability of using nonhuman animals in research. The articles in this issue assess how moral argument and empirical studies stand to guide animal research policies and practices in future years.

Many in bioethics have come to regard issues of animal research as a subfield of research ethics, bringing it closer to human research ethics. Animal ethics, like public health ethics, has struggled for recognition in bioethics. As the contributions to this issue show, some in bioethics who initially focused on human research ethics have now devoted significant time to animal research ethics, and some who started

\footnotetext{
T. L. Beauchamp ( $₫)$

Department of Philosophy and Kennedy Institute of Ethics, Georgetown University, 425 Healy

Bldg, Washington, DC 20057, USA

e-mail: beauchat@georgetown.edu

H. R. Ferdowsian

Department of Medicine, The George Washington University, 2150 Pennsylvania Avenue,

Washington, DC 20037, USA

e-mail: hferdowsian@mfa.gwu.edu

J. P. Gluck

Department of Psychology, University of New Mexico, Albuquerque, NM 87131, USA

e-mail: jgluck@unm.edu

J. P. Gluck

Kennedy Institute of Ethics, Georgetown University, Washington, DC 20057, USA
} 
in animal research ethics have now joined the larger bioethics community. Common interests in human and animal research ethics are indicative of changing views of nonhuman animals, their status in society, and human obligations to nonhuman animals.

Public policy in various government agencies has recently come to reflect some of these changes. Between late 2011 and mid 2013, an unprecedented shift occurred at the US National Institutes of Health (NIH) in animal research policy. Following a public controversy and a 2010 congressional request, the NIH commissioned the formation of an Institute of Medicine (IOM) Committee on the Use of Chimpanzees in Biomedical and Behavioral Research, which released its final report on December 15, 2011 [2]. The committee concluded that chimpanzees are now largely unnecessary for biomedical research, and it recommended a set of restrictive guiding principles and criteria for the assessment of current and future uses of chimpanzees in biomedical and behavioral research. In a range of cases the committee recommended that chimpanzee "acquiescence" (voluntary acceptance) be required for research participation [2, pp. 6, 69].

This report was adopted as public policy within two hours of its release [3], followed by the appointment of an NIH Council of Councils Working Group to configure implementation of the report [4]. On June 26, 2013, NIH adopted almost all of the working group's demanding recommendations and announced that it planned to release the majority of federally owned or sponsored chimpanzees to sanctuaries in the United States [5]. In effect, NIH declared that it was phasing out invasive chimpanzee research. Concurrently, the United States Fish and Wildlife Service released its proposal to list captive chimpanzees as endangered, similar to chimpanzees living in the wild [6]. If adopted as policy, listing all chimpanzees as endangered would prohibit or limit invasive research conducted on publicly and privately owned chimpanzees. The United States would join the majority of countries across the globe that ban or significantly restrict invasive research on great apes.

These developments in animal research policy provide new opportunities to reflect on the ethics of nonhuman animal use at a critical point in the histories of both animal research ethics and bioethics. Paradoxically, there seems to be a broadening gap between rapid advances made in scientific knowledge about nonhuman animals and guidelines that apply to their use in research. Many guidelines appear to have outdated assumptions and policies, though some have recently made considerable advances.

The seven articles in this issue challenge various longstanding assumptions about nonhuman animals in light of both scientific advances and progress in bioethics. The articles address traditional notions of the nature of animals, how nonhuman animals are used in research, and whether concepts historically reserved for human research can be usefully applied to decisions about animal research. Authors explore how major concepts in human research ethics, such as autonomous decision making, refusal of participation, assent, obligations of avoiding harm, and demands of justice can and should be applied to research involving nonhuman animals. Examples of nonhuman primates are several times used in these articles to address the adequacy 
of current animal research guidelines, recent animal research findings, new inquiries into animal minds, and the like.

In the first article, "Lessons Learned: Challenges in Applying Current Constraints on Research on Chimpanzees to Other Animals" [7], Jeffrey Kahn examines the conclusions of the IOM Committee's work on the necessity of the use of chimpanzees in biomedical and behavioral research. As the IOM committee's chair, he examines key features of the committee's work and its implications. Kahn explores the constraints that were placed on the moral deliberations that the committee could undertake. He describes how ethics informed the committee's process and findings, despite the committee's narrow mandate from the NIH to examine only the "scientific necessity" of chimpanzee research. He also describes ways in which the committee introduced novel concepts for animal research guidelines, including requirements for chimpanzee acquiescence and higher standards of ethologically appropriate environments. He notes that the use of nonhuman animals is often determined by their availability and that criteria based merely on scientific necessity force into the open issues of moral justification. As the only member trained in ethics on the IOM committee, Kahn provides a view of lessons learned through this committee's bold and critically important deliberations and findings.

In "Moving Beyond the Welfare Standard of Psychological Well-Being for Nonhuman Primates" [8], John Gluck examines late-twentieth-century US federal policy, especially the critical transition from the 1985 amendment to the Animal Welfare Act, which promoted the psychological well-being of nonhuman primates, to the 2011 IOM committee's requirement for ethologically appropriate environments. Gluck analyzes the historical shift from an emphasis on hygienic environments and "clean cages" with "enrichment" tools to a requirement that chimpanzees experience living conditions approximating those in the wild. Gluck assesses the public controversy, economic considerations, legal mandates, and the evolution of ethical concerns. From his personal history as a primate investigator, Gluck appraises the various determinants of chimpanzee well-being and envisions what recent changes made in policy governing chimpanzee research could mean for other nonhuman primates.

Whereas Gluck focuses on psychological well-being, Tom Beauchamp and Victoria Wobber, in "Autonomy in Chimpanzees" [9], use evidence about the psychological capacities of chimpanzees to examine how concepts such as understanding, voluntariness, and autonomy should be understood. Noting that literature on the mental capacities and cognitive mechanisms of great apes has not explored the subject of autonomy, they provide a theory of autonomy buttressed by data from psychological research on chimpanzees. They argue that chimpanzees have the capacity to act autonomously, and often do, by gathering information and making choices in remarkably sophisticated ways. Beauchamp and Wobber provide a concise theory of autonomy as self-initiated action that is intentional, adequately informed, and free of controlling influences. They describe how chimpanzees meet these requirements, based on the available literature on the cognitive capacities of chimpanzees. They then assess leading theories of autonomy, rejecting them on 
grounds that these theories are too narrow and demanding to adequately express the basic conditions of autonomy for either human or nonhuman animals.

In "Chimpanzees as Vulnerable Subjects in Research" [10], Jane Johnson and Neal Barnard examine the cognitive capacities of chimpanzees and how their vulnerabilities sometimes result from diminished mental capacities, including those caused in research settings. They assess how these facts should affect decisions about the use of these animals in research. They draw from recent studies of primate cognition and widely invoked bioethical principles to demonstrate how inherent and situational factors can diminish capacity in chimpanzees in research settings, and sometimes cause psychological disorders diagnosable by using human clinical criteria. Citing historical differences between human research and animal research, Johnson and Barnard examine how principles of justice and the vulnerability of chimpanzees as a class are relevant considerations. They argue that chimpanzees should be treated in ways closely analogous to the ways in which vulnerable human subjects are treated in research. They propose additional safeguards such as thirdparty consent. Many of the arguments presented by Johnson and Barnard are applicable across species and could have implications for assessments about animal research generally.

In "Harms and Deprivation of Benefits for Nonhuman Primates Used in Research" [11], Hope Ferdowsian and Agustín Fuentes argue that the risks of harm to and the absence of benefits for nonhuman primates are morally important considerations when judging whether their use in research is justified. They contend that risk in nonhuman primate research should be modeled on the use of risk thresholds in human research, for example, as used in research with children. They contrast a relative inattention to harm-causing activities in animal research with the high level of attention paid to them in human research. They then propose and defend a risk threshold standard for nonhuman primates in which these individuals should not be exposed to risks that exceed those posed in the daily lives of healthy primates living in safe species-appropriate environments. They maintain that the majority of current invasive biomedical and behavioral research exceeds this standard and that the majority of laboratory research involves a high or certain risk of significant physical, psychological and social harm. Rather than focusing on risk mitigation, Ferdowsian and Fuentes emphasize the importance of assessing the full range of determinants of well-being in nonhuman primates.

In "Should Protections for Research with Humans Who Cannot Consent Apply to Research with Nonhuman Primates?" [12], David Wendler probes the ethical and practical implications of applying regulations used in human pediatric research to decisions about nonhuman primate research. Drawing from his experience with human populations who are unable to provide valid consent, Wendler examines how research that is not beneficial for subjects can be ethically conducted and justified in these settings. He argues that several protections for research with humans who cannot consent would be valuable_-including low risk thresholds, the potential to discover highly valuable information, surrogate consent on behalf of individual subjects, and assent by the subjects. These conditions of justified research deserve consideration, he argues, for research with nonhuman primates. He examines the extent to which concepts and normative judgments found in human research 
protections, such as social value, subject selection, acceptable risks, exceptional cases, independent review, and dissent, can be usefully deployed in decisions about nonhuman primate research.

In "Confronting Ethical Permissibility in Animal Research" [13], Chong Un Choe Smith makes an assessment of what she calls a common assumption in animal research, which is that when risks are unjustifiably high for use in human populations, it is justifiable to use nonhuman animal subjects as long as there is a scientific necessity for doing so. She contends that this common assumption raises critical questions about the justification of proceeding and about when it is permissible to continue with the research. She maintains that using nonhuman animal subjects is often inconsistent with one or more principles of justice that are fundamental to human research ethics and appropriate in animal research. One such principle requires that specifiable classes of individuals not be subjected to a disproportionate share of the burdens or risks of research. Choe Smith extends this principle to nonhuman animals and argues that the principle is violated if nonhuman animals bear an inordinate share of the risks of research while failing to procure any benefits. Choe Smith also argues that the utilitarian justifications commonly used to justify animal research fail.

As guest editors of this special issue of Theoretical Medicine and Bioethics, we wish to thank the authors for their commitment to this issue and the editors of the journal for their deep interest in the timely subject of animal research ethics.

\section{References}

1. DeGrazia, David. 2006. Regarding animals: Mental life, moral status, and use in biomedical research: An introduction to the special issue. Theoretical Medicine and Bioethics 27: 277-284.

2. Institute of Medicine. 2011. Chimpanzees in biomedical and behavioral research: Assessing the necessity. Washington, DC: National Academies Press.

3. National Institutes of Health. 2011. Statement by NIH Director Dr. Francis Collins on the Institute of Medicine report addressing the scientific need for the use of chimpanzees in research. Office of the Director (December 15, 2011). http://www.nih.gov/news/health/dec2011/od-15.htm. Accessed July $11,2013$.

4. Council of Councils Working Group on the Use of Chimpanzees in NIH-Supported Research. 2013. Report. http://dpcpsi.nih.gov/sites/default/files/FNL_Report_WG_Chimpanzees_0.pdf. Accessed July 30, 2013.

5. National Institutes of Health. 2013. Announcement of agency decision: Recommendations on the use of chimpanzees in NIH-supported research. http://dpcpsi.nih.gov/council/pdf/NIH_response_to_ Council_of_Councils_recommendations_62513.pdf. Accessed July 10, 2013.

6. U.S. Fish and Wildlife Service. 2013. U.S. Fish and Wildlife Service proposes protection for all chimpanzees-captive and wild-as endangered. Office of External Affairs (June 11, 2013). http:// www.fws.gov/home/newsroom/serviceproposeschimpanzeesNR06112013.html. Accessed July 10, 2013.

7. Kahn, Jeffrey. 2014. Lessons learned: Challenges in applying current constraints on research on chimpanzees to other animals. Theoretical Medicine and Bioethics. doi:10.1007/s11017-014-9284-6.

8. Gluck, John. 2014. Moving beyond the welfare standard of psychological well-being for nonhuman primates. Theoretical Medicine and Bioethics. doi:10.1007/s11017-014-9289-1.

9. Beauchamp, Tom, and Victoria Wobber. 2014. Autonomy in chimpanzees. Theoretical Medicine and Bioethics. doi:10.1007/s11017-014-9287-3. 
10. Johnson, Jane, and Neal Barnard. 2014. Chimpanzees as vulnerable subjects in research. Theoretical Medicine and Bioethics. doi:10.1007/s11017-014-9286-4.

11. Ferdowsian, Hope, and Agustín Fuentes. 2014. Harms and deprivation of benefits for nonhuman primates used in research. Theoretical Medicine and Bioethics. doi:10.1007/s11017-014-9288-2.

12. Wendler, David. 2014. Should protections for research with humans who cannot consent apply to research with nonhuman primates? Theoretical Medicine and Bioethics. doi:10.1007/s11017-0149285-5.

13. Choe Smith, Chong Un. 2014. Confronting ethical permissibility in animal research. Theoretical Medicine and Bioethics. doi:10.1007/s11017-014-9290-8. 